\title{
Attraction of the sand fly Nyssomyia neivai (Diptera: Psychodidae) to chemical compounds in a wind tunnel
}

\author{
Vicente Estevam Machado ${ }^{1}$, Arlene Gonçalves Corrêa², Thais Marchi Goulart ${ }^{3}$, Flávia Benini da Rocha Silva', \\ Dennys Ghenry Samillan Ortiz ${ }^{4}$ and Mara Cristina Pinto ${ }^{1 *}$
}

\begin{abstract}
Background: Similar to other hematophagous insects, male and female sand flies must feed on plants to obtain sugar and, subsequently, energy to complete their life cycles. A large number of compounds emitted by plants may act as volatile signals to these insects. Primary alcohols have been detected in some plants, but in small amounts. In a previous report, the attractiveness of saturated primary alcohols with 7 to 9 carbons was evaluated for Lutzomyia longipalpis, the vector of American visceral leishmaniasis, with positive results.
\end{abstract}

Methods: In the present study, a wide range of primary alcohols, 3 to 10 carbons, were tested to investigate their attractiveness to another sand fly species, Nyssomyia neivai, a putative vector of American cutaneous leishmaniasis. The mixture of compounds that induced the best sand fly response was also evaluated.

Results: Of the eight compounds evaluated, hexanol and octanol elicited the best attractive responses for sand fly females.

Conclusion: Phytochemicals may be an interesting source of search for new sand fly attractants.

Keywords: Sand flies, Wind tunnel, Attractiveness, Alcohols, Plant volatiles, Olfactometry, Nyssomyia neivai, Octanol, Hexanol

\section{Background}

Generally, the concept of kairomones for hematophagous insects is related to host volatiles, and the development of new lures is focused on those compounds. However, considering that hematophagous insects also feed on plant tissues, phytochemical attractants are very interesting targets, but less studied compared with kairomones from vertebrate animals [1].

There have been behavioural reports on the attractiveness of floral odours or single floral compounds to mosquitoes [2-4]. For sand flies, studies with plants [5-7] fruits [8] and honey [9] have shown their effective roles as attractants, but there has been no isolation of compounds from these different sources.

\footnotetext{
* Correspondence: marap@fcfar.unesp.br

'Departamento de Ciências Biológicas, Faculdade de Ciências Farmacêuticas, Universidade Estadual Júlio de Mesquita Filho, UNESP, 14801-902 Araraquara, SP, Brazil

Full list of author information is available at the end of the article
}

In a previous study conducted in a wind tunnel, the attractiveness of the host kairomone 1-octen-3-ol was compared with three saturated primary alcohols (1heptanol, 1-octanol and 1-nonanol) for the sand fly Lutzomyia longipalpis [10]. Those saturated primary alcohols are present in small quantities in some plant volatiles. The results demonstrated a relative attractive response of female L. longipalpis to 1-heptanol and 1nonanol. Such results indicate that other saturated primary alcohols may also be attractive to sand fly species. The present study investigated the attractiveness of a broad range of saturated primary alcohols (3 to 10 carbons) for Nyssomyia neivai, a putative vector of cutaneous leishmaniasis in South America [11]. The compounds that evoked the best responses were combined and evaluated for their attractiveness. 


\section{Methods}

\section{Sand flies and laboratory maintenance}

Insects were collected, using manual aspiration on the wall of one house located at the edge of the Mogi-Guaçu River, northeast center of São Paulo State, Brazil (21 ${ }^{\circ} 35^{\prime}$ $\left.13^{\prime} \mathrm{S} 48^{\circ} 04^{\prime} 15^{\prime} \mathrm{W}\right)$. In the laboratory, the insects were maintained in netting cages with a $30 \%$ solution of sucrose fed ad libitum under controlled conditions $\left(26 \pm 1^{\circ} \mathrm{C}, 80-\right.$ 90\%, 12:12 (L:D) photoperiod).

\section{Experiments in the wind tunnel}

The experiments were performed in a wind tunnel (previously described) [12] within three to four days after the field collection of insects. Tests were performed from 9:00 to 19:00 and, at the end of the assay, the insects were identified according to the classification proposed by Galati [13].

Groups of three females were placed in a releasing chamber, for a total of 30 different insects for each concentration of the evaluated compounds. The releasing chambers were placed in the wind tunnel $50 \mathrm{~cm}$ from the stimulus. Tests lasted for $2 \mathrm{~min}$, and two behaviours were evaluated: activation and attraction. Activation was defined as the number of sand flies that left the releasing chamber, and attraction was defined as the number of sand flies that reached the stimulus.

The compounds evaluated were: 1-propanol (99.5\%), 1-butanol (99.4\%), 1-pentanol (>99.5\%), 1-hexanol (98\%), 1-heptanol (98\%), 1-octanol (>99\%), 1- nonanol (98\%) and 1-decanol (>99\%) (hereafter propanol, butanol, pentanol, hexanol, heptanol, octanol, nonanol and decanol) (Sigma-Aldrich). The compound 1-octen3-ol (hereafter octenol) has been already shown to be attractive to $N$. neivai [12], so it was used as a positive control. All compounds were diluted in hexane and tested at concentrations of $10 \%, 50 \%$ and $100 \%$ (neat). When necessary, a lower concentration (5\%) was also used. Each compound was delivered $(200 \mu \mathrm{L})$ on a filter paper $(4 \times 4 \mathrm{~cm})$ in the entrance of the tunnel. The control was hexane presented the same way prior to testing the other compounds.

After these tests, the three compounds with the best responses (heptanol, octanol, and nonanol) were combined (1:1:1) and subsequently evaluated in the wind tunnel.

The interval between a compound and another was at least two hours, when the tunnel was cleaned with hexane and allowed to dry.

\section{Statistical analysis}

Chi square tests were used to evaluate the different proportions of sand fly females activated and attracted by each compound. Initially, the tests were conducted for all concentrations simultaneously. If a significant difference was verified, each of the two groups was compared separately. The statistical analyses were performed using BioEstat (available in http://www.mamiraua.org.br/pt-br/ downloads/programas/bioestat-versao-53/).

\section{Results}

Considering the attraction behaviour, sand flies responded in a clear dose-dependent manner to pentanol, hexanol, octanol and octenol. Propanol and butanol were not significantly different from the control for activation (propanol $\mathrm{X}^{2}=6.9, \mathrm{df}=3, \mathrm{P}=0.07$; butanol $\mathrm{X}^{2}=6.7 \mathrm{df}=3$, $\mathrm{P}=0.1$ ) or attraction (propanol $\mathrm{X}^{2}=1.1, \mathrm{df}=3, \mathrm{P}=0.8$; butanol $X^{2}=7.6, d f=3, P=0.1$ ). The other evaluated compounds presented some degree of activation (pentanol, $\mathrm{X}^{2}=32.9, \mathrm{df}=3, \mathrm{P}<0.0001$; hexanol $\mathrm{X}^{2}=68.7, \mathrm{df}=3$, $\mathrm{P}<0.0001$; heptanol $\mathrm{X}^{2}=47.7, \mathrm{df}=3, \mathrm{P}<0.0001$; octanol $\mathrm{X}^{2}=60.6 \mathrm{df}=3, \mathrm{P}<0$ 0.0001; nonanol $\mathrm{X}^{2}=46.3$, $\mathrm{df}=3, \mathrm{P}<0.0001$; decanol $\left.\mathrm{X}^{2}=21.7, \mathrm{df}=3, \mathrm{P}<0.0001\right)$ or attraction (pentanol, $\mathrm{X}^{2}=41.6, \mathrm{df}=3, \mathrm{P}<0.0001$; hexanol $\mathrm{X}^{2}=50.6, \mathrm{df}=3, \mathrm{P}<0.0001$; heptanol $\mathrm{X}^{2}=$ 13.8, df $=3, \mathrm{P}=0.008$; octanol $\mathrm{X}^{2}=37.9 \mathrm{df}=3, \mathrm{P}<0.0001$; nonanol $\mathrm{X}^{2}=22.7, \mathrm{df}=3, \mathrm{P}<0.0001$; decanol $\mathrm{X}^{2}=11.3$, $\mathrm{df}=3, \mathrm{P}=0.01$ ) for females $N$. neivai sand flies (Table 1 ).

With octenol as a positive control and $70 \%$ used as a cut off for responses, pentanol, hexanol, heptanol, octanol, nonanol and, to a lesser extent, decanol, elicited activation behaviours. However, according to the same criteria, hexanol and octanol were the best attractants for sand fly females. Octanol was even better than octenol at lower concentrations (50\% and 10\%).

The concentration of $5 \%$ was added to trials with heptanol, octanol and nonanol because they elicited high responses of activation at $10 \%$. Those compounds were mixed (1:1:1) and tested again. The results showed that $93 \%$ of females were activated and $63 \%$ were attracted by the mixture. There was an improvement in activation that was not reflected for attraction.

\section{Discussion}

Our study compared the attractiveness of saturated primary alcohols to sand flies with 1-octen-3-ol, an unsaturated secondary alcohol, used as lure for hematophagous insects. The results emphasised that, similar to a previous study with the sand fly species L. longipalpis [10], such compounds also act as activators and/or attractants for $N$. neivai. The results are also in agreement with our prior survey [12] and showed that flies taken directly from the field can be used in laboratory olfactometer experiments, in spite of different physiological conditions, because they showed consistently higher activation and attraction responses to the evaluated compounds than to air control.

Octenol is a known host kairomone found in cattle [14], human breath [15] and human skin emanation [16]. For sand flies, octenol has been shown to elicit a response in L. longipalpis in electrophysiological 
Table 1 Percentages of activated and attracted $N$. neivai females to individual alcohols and mixture of three alcohols ( $\mathrm{n}=\mathbf{3 0}$ for each compound)

\begin{tabular}{|c|c|c|}
\hline Compound & Activated (\%) & Attracted (\% \\
\hline Control & $13 \mathrm{a}$ & $7 a$ \\
\hline Propanol 10\% & $7 a$ & $7 \mathrm{a}$ \\
\hline Propanol 50\% & $7 a$ & $3 a$ \\
\hline Propanol 100\% & $27 \mathrm{a}$ & $10 \mathrm{a}$ \\
\hline Control & 13 a & $7 \mathrm{a}$ \\
\hline Butanol 10\% & $27 \mathrm{a}$ & $10 \mathrm{a}$ \\
\hline Butanol 50\% & $30 \mathrm{a}$ & $13 \mathrm{a}$ \\
\hline Butanol 100\% & $43 a$ & $30 \mathrm{a}$ \\
\hline Control & $13 a$ & $7 \mathrm{a}$ \\
\hline Pentanol 10\% & $40 a b$ & $10 \mathrm{a}$ \\
\hline Pentanol 50\% & $70 \mathrm{bc}$ & $63 \mathrm{~b}$ \\
\hline Pentanol 100\% & $80 \mathrm{c}$ & $67 \mathrm{~b}$ \\
\hline Control & $13 \mathrm{a}$ & $7 \mathrm{a}$ \\
\hline Hexanol 10\% & $30 \mathrm{a}$ & $17 \mathrm{a}$ \\
\hline Hexanol 50\% & $90 \mathrm{~b}$ & $57 \mathrm{~b}$ \\
\hline Hexanol 100\% & $100 \mathrm{~b}$ & $87 \mathrm{c}$ \\
\hline Control & $13 \mathrm{a}$ & $7 \mathrm{a}$ \\
\hline Heptanol 5\% & $53 \mathrm{~b}$ & $47 \mathrm{~b}$ \\
\hline Heptanol 10\% & $83 \mathrm{~b}$ & $43 b$ \\
\hline Heptanol 50\% & 87 bc & $40 \mathrm{~b}$ \\
\hline Heptanol 100\% & $77 \mathrm{~b}$ & $33 a$ \\
\hline Control & $13 a$ & $7 \mathrm{a}$ \\
\hline Octanol 5\% & $77 \mathrm{~b}$ & $57 \mathrm{~b}$ \\
\hline Octanol 10\% & $80 \mathrm{~b}$ & $70 \mathrm{~b}$ \\
\hline Octanol 50\% & $83 \mathrm{~b}$ & $70 \mathrm{~b}$ \\
\hline Octanol 100\% & $97 \mathrm{~b}$ & $73 \mathrm{~b}$ \\
\hline Control & $13 a$ & $7 \mathrm{a}$ \\
\hline Nonanol 5\% & $70 \mathrm{~b}$ & $53 \mathrm{~b}$ \\
\hline Nonanol 10\% & $87 \mathrm{~b}$ & $43 b$ \\
\hline Nonanol 50\% & $83 \mathrm{~b}$ & $63 b$ \\
\hline Nonanol 100\% & $70 \mathrm{~b}$ & $47 b$ \\
\hline Control & $13 \mathrm{a}$ & $7 \mathrm{a}$ \\
\hline Decanol 10\% & $40 \mathrm{a}$ & $20 \mathrm{a}$ \\
\hline Decanol 50\% & $70 \mathrm{~b}$ & $37 b$ \\
\hline Decanol 100\% & $57 \mathrm{~b}$ & $40 \mathrm{~b}$ \\
\hline Control & $13 \mathrm{a}$ & $7 \mathrm{a}$ \\
\hline Octenol 10\% & $13 a$ & $10 \mathrm{a}$ \\
\hline Octenol 50\% & $73 \mathrm{~b}$ & $47 \mathrm{~b}$ \\
\hline Octenol 100\% & $80 \mathrm{~b}$ & $80 \mathrm{bc}$ \\
\hline Mixture* & 97 & 63 \\
\hline
\end{tabular}

For each compound, different letters within a column indicate significant differences $(P<0.05)$. Bold signs indicates values $\geq 70 \%$; a cut off for responses based on octenol. *Mixture $=$ heptanol, octanol and nonanol (1:1:1). recordings [17] and attractive behaviours in a wind tunnel [10]. For Nyssomyia intermedia and N. neivai, octenol also elicited attractive responses in field studies $[18,19]$. In addition to its occurrence in mammals, octenol is also identified in many studies with volatile organic compounds (VOC) in different species of plants such as watermelon [20], flowers of lucerne [21] and mango [22].

The alcohols used in the present study are not host kairomones, but they are present in plant volatiles. For example, octanol and hexanol are present in watermelon [20] and strawberry [23]; and butanol, pentanol, hexanol, and octanol are present in guava [24]. Considering that sand flies require sugar meals from plants to survive, those compounds could be considered to be phytochemical attractants.

Previous reports have demonstrated the attractiveness of plants to sand flies $[7,8,25]$. A laboratory study in a wind tunnel evaluated unifloral honey odours as attractants for different populations of three sand flies species, and the responses were species and population specific [9].

Despite the evidence of plant attractiveness to sand flies, there is a gap in the identification of phytochemical attractants isolated from the plants. The main advantage of phytochemical attractants for hematophagous insects is that they lure males and females of all ages and females in all gonotrophic states [1]. Our results show that, in addition to host kairomones, other compounds, such as phytochemical, should also be investigated as attractants for sand flies.

\section{Conclusions}

The compounds hexanol and octanol elicited the best attractive responses for $N$. neivai females in bioassays. The next step is to evaluate if those compounds will be attractive for other sand fly species in field.

Competing interests

The authors declare that they have no competing interests.

\section{Authors' contributions}

VM, MP and AC conceived and executed the study and drafted the manuscript. VM, TG and FS conducted field captures of sandflies and bioassay experiments. DO contributed to field captures and the manuscript. All authors read and approved the final manuscript.

\section{Acknowledgements}

MP received a grant (F-4587-1) from the International Foundation for Science (IFS) to build the wind tunnel and financial support for publication from Programa de Apoio ao Desenvolvimento Científico da Faculdade de Ciências Farmacêuticas at UNESP (PADC/FCFAr).

\section{Author details}

'Departamento de Ciências Biológicas, Faculdade de Ciências Farmacêuticas, Universidade Estadual Júlio de Mesquita Filho, UNESP, 14801-902 Araraquara, SP, Brazil. ${ }^{2}$ Departamento de Química, Universidade Federal de São Carlos,

13565-905 São Carlos, SP, Brazil. ${ }^{3}$ Departamento de Zoologia Animal, Universidade Estadual de Campinas, 13083-970 Campinas, SP, Brazil. ${ }^{4}$ Universidade de Franca, 14404-611 Franca, SP, Brazil. 
Received: 8 November 2014 Accepted: 17 February 2015

Published online: 07 March 2015

\section{References}

1. Foster WA. Phytochemicals as population sampling lures. J Am Mosq Control Assoc. 2008;24(1):138-46.

2. Healy TP, Jepson PC. The location of floral nectar sources by mosquitoes: the long-range responses of Anopheles arabiensis Patton (Diptera: Culicidae) to Achillea millefolium flowers and isolated floral odour. Bull Entomol Res. 1988;78(04):651-7.

3. Jhumur US, Dotterl S, Jurgens A. Floral odors of Silene otites: their variability and attractiveness to mosquitoes. J Chem Ecol. 2008;34(1):14-25.

4. Otienoburu PE, Ebrahimi B, Phelan PL, Foster WA. Analysis and optimization of a synthetic milkweed floral attractant for mosquitoes. J Chem Ecol. 2012:38(7):873-81.

5. Schlein Y, Muller G. Assessment of plant tissue feeding by sand flies (Diptera: Psychodidae) and mosquitoes (Diptera: Culicidae). J Med Entomol. 1995;32(6):882-7.

6. Schlein Y, Jacobson RL, Muller GC. Sand fly feeding on noxious plants: a potential method for the control of leishmaniasis. Am J Trop Med Hyg. 2001;65(4):300-3.

7. Muller GC, Revay EE, Schlein Y. Relative attraction of the sand fly Phlebotomus papatasi to local flowering plants in the Dead Sea region. J Vector Ecol. 2011;36 Suppl 1:S187-94.

8. Junnila A, Muller GC, Schlein Y. Attraction of Phlebotomus papatasi to common fruit in the field. J Vector Ecol. 2011;36 Suppl 1:S206-11.

9. Wasserberg G, Kirsch P, Rowton ED. Orientation of colonized sand flies Phlebotomus papatasi, P. duboscai, and Lutzomyia longipalpis (Diptera: Psychodidae) to diverse honeys using a 3-chamber in-line olfactometer. J Vector Ecol. 2014;39(1):94-102.

10. Magalhaes-Junior J, Barrouin-Melo S, Correa A, da Rocha Silva F, Machado V, Govone J, et al. A laboratory evaluation of alcohols as attractants for the sandfly Lutzomyia longipalpis (Diptera:Psychodidae). Parasit Vectors. 2014;7(1):60.

11. Cordoba-Lanus E, De Grosso ML, Pinero JE, Valladares B, Salomon OD. Natural infection of Lutzomyia neivai with Leishmania spp. in northwestern Argentina. Acta Trop. 2006;98(1):1-5.

12. Pinto MC, Bray DP, Eiras AE, Carvalheira HP, Puertas CP. Attraction of the cutaneous leishmaniasis vector Nyssomyia neivai (Diptera: Psychodidae) to host odour components in a wind tunnel. Parasit Vectors. 2012;5:210.

13. Galati EAB. Classificaçâo de Phlebotominae. In: Rangel EF, Lainson R, editors. Flebotomíneos do Brasil. Rio de Janeiro, Brazil: Fiocruz; 2003.

14. Hall DR, Beevor PS, Cork A, Nesbitt BF, Vale GA. 1-Octen-3-ol. A potent olfactory stimulant and attractant for tsetse isolated from cattle odours. Int J Trop Insect Sci. 1984;5(Special Issue 05):335-9.

15. Xue R, Dong L, Zhang S, Deng C, Liu T, Wang J, et al. Investigation of volatile biomarkers in liver cancer blood using solid-phase microextraction and gas chromatography/mass spectrometry. Rapid Commun Mass Spectrom. 2008;22(8):1181-6.

16. Bernier UR, Kline DL, Barnard DR, Schreck CE, Yost RA. Analysis of human skin emanations by gas chromatography/mass spectrometry. 2. Identification of volatile compounds that are candidate attractants for the yellow fever mosquito (Aedes aegypti). Anal Chem. 2000;72(4):747-56.

17. Sant'ana AL, Eiras AE, Cavalcante RR. Electroantennographic responses of the Lutzomyia (Lutzomyia) longipalpis (Lutz \& Neiva) (Diptera: Psychodidae) to 1-octen-3-ol. Neotrop Entomol. 2002;31:13-7.

18. Andrade AJ, Andrade MR, Dias ES, Pinto MC, Eiras AE. Are light traps baited with kairomones effective in the capture of Lutzomyia longipalpis and Lutzomyia intermedia? An evaluation of synthetic human odor as an attractant for phlebotomine sand flies (Diptera: Psychodidae: Phlebotominae). Mem Inst Oswaldo Cruz. 2008;103(4):337-43.

19. Pinto MC, Barbieri K, Silva MC, Graminha MA, Casanova C, Andrade AJ, et al. Octenol as attractant to Nyssomyia neivai (Diptera:Psychodidae: Phlebotominae) in the field. J Med Entomol. 2011;48(1):39-44.

20. Yajima I, Sakakibara H, Ide J, Yanai T, Kazuo H. Volatile flavor components of watermelon (Citrullus vulgaris). Agric Biol Chem. 1985;49(11):3145-50.

21. Tava A, Pecetti L. Volatiles from Medicago sativa complex flowers. Phytochemistry. 1997;45(6):1145-8.
22. Kamala Jayanthi PD, Kempraj V, Aurade RM, Venkataramanappa RK, Nandagopal B, Verghese A, et al. Specific volatile compounds from mango elicit oviposition in gravid Bactrocera dorsalis females. J Chem Ecol. 2014;40(3):259-66.

23. Hamilton-Kemp TR, Loughrin $J H$, Andersen RA. Identification of some volatile compounds from strawberry flowers. Phytochemistry. 1990;29(9):2847-8.

24. Pino JA, Bent L. Odour-active compounds in guava (Psidium guajava L. CV. Red Suprema). J Sci Food Agric. 2013;93(12):3114-20.

25. Schlein Y, Yuval B. Leishmaniasis in the Jordan Valley. IV. Attraction of Phlebotomus papatasi (Diptera: Psychodidae) to plants in the field. J Med Entomol. 1987;24(1):87-90.

\section{Submit your next manuscript to BioMed Central and take full advantage of:}

- Convenient online submission

- Thorough peer review

- No space constraints or color figure charges

- Immediate publication on acceptance

- Inclusion in PubMed, CAS, Scopus and Google Scholar

- Research which is freely available for redistribution 